\title{
Solvability of initial-boundary value problem of a multiple characteristic fifth-order operator-differential equation
}

\author{
Nashat Faried ${ }^{1}$, Labib Rashed ${ }^{1}$, Abdel Baset I. Ahmed ${ }^{2 *}$ and Mohamed A. Labeeb ${ }^{3}$
}

${ }^{*}$ Correspondence:

abdel2007@yandex.ru

${ }^{2}$ Helwan University, 11865 Cairo, Egypt

Full list of author information is available at the end of the article

\begin{abstract}
In this study, we establish existence-uniqueness of a vector function in appropriate Sobolev-type space for a boundary value problem of a fifth-order operator differential equation. Proper conditions are obtained for the given problem to be well-posed. Much effort is devoted to develop the association between these conditions and the operator coefficients of the investigated equation. In this paper, accurate estimates of the norms of the intermediate derivatives operators are presented and used to determine the solvability conditions.
\end{abstract}

Keywords: Initial-boundary value problems, Operator-differential equation, Self-adjoint operator, Intermediate derivative operator

Mathematical Subject Classifications 2010: 34A12, 34G10, 34k10, 35J40, 47D03

\section{Introduction}

Initial-boundary value problem theory in Banach or Hilbert space on the real axis is useful because it enables to study equations of parabolic and elliptic differential operators with initial-boundary conditions.

We shall study the following initial-boundary value problem in a separable Hilbert space $H$ :

$$
\begin{aligned}
& \left(-\frac{d^{2}}{d x^{2}}+A^{2}\right)\left(\frac{d}{d x}+A\right)^{3} u(x)+\sum_{j=1}^{5} A_{j} \frac{d^{5-j}}{d x^{5-j}} u(x)=f(x), \\
& x \in R=(-\infty,+\infty) \\
& \frac{d^{s} u(0)}{d x^{s}}=0, s=0,1,2,3
\end{aligned}
$$

where $A$ is a self-adjoint positively defined operator and $A_{j}, j=1,2,3,4,5$ are linear unbounded operators. From now on, the derivatives are accepted through the distributions theory (see [1]). We specify the following subspaces.

We consider $f(x) \in L_{2}(R ; H)$, and $u(x) \in W_{2}^{5}(R ; H)$, where:

$$
L_{2}(R ; H)=\left\{f(x):\|f(x)\|_{L_{2}(R ; H)}=\left(\int_{-\infty}^{+\infty}\|f(x)\|_{H}^{2} d x\right)^{\frac{1}{2}}<+\infty\right\},
$$

(c) The Author(s). 2019 Open Access This article is distributed under the terms of the Creative Commons Attribution 4.0 International License (http://creativecommons.org/licenses/by/4.0/), which permits unrestricted use, distribution, and reproduction in any medium, provided you give appropriate credit to the original author(s) and the source, provide a link to the Creative Commons license, and indicate if changes were made. 


$$
\begin{aligned}
& W_{2}^{5}(R ; H)=\left\{u(t): \frac{d^{5} u(x)}{d x^{5}} \in L_{2}(R ; H), A^{5} u(x) \in L_{2}(R ; H)\right\} . \text { With the norm } \\
& \|u\|_{W_{2}^{5}(R ; H)}=\left(\left\|\frac{d^{5} u}{d x^{5}}\right\|_{L_{2}(R ; H)}^{2}+\left\|A^{5} u\right\|_{L_{2}(R ; H)}^{2}\right)^{\frac{1}{2}}(\text { see }[2-5]) .
\end{aligned}
$$

Definition 1 If for any $f(x) \in L_{2}(R ; H)$, there exists a vector function $u(x) \in W_{2}^{5}(R ; H)$ that satisfies (1.1) almost everywhere in $R$, then it is known as a regular solution of (1.1)

Definition 2 If for any function $f(x) \in L_{2}(R ; H)$, there exists a regular solution $u(x) \in$ $W_{2}^{5}(R ; H)$ of (1.1) satisfying the initial boundary conditions (1.2) in the sense that

$$
\lim _{x \rightarrow 0}\left\|A^{\frac{9}{2}-i} \frac{d^{i} u(x)}{d x^{i}}\right\|_{H}=0, \quad i=0,1,2,3
$$

and the inequality

$$
\|u\|_{W_{2}^{5}(R ; H)} \leq \text { const }\|f\|_{L_{2}(R ; H)},
$$

holds, then problems (1.1) and (1.2) will be regularly solvable (see [6, 7]).

\section{Main results}

From the theorem of intermediate derivatives (see [8, 9]) if $u(x) \in W_{2}^{5}(R ; H)$, then $A^{5-j} \frac{d^{j} u(x)}{d x^{j}} \in L_{2}(R ; H), j=\overline{1,5}$ and the following inequalities:

$$
\left\|A^{5-j} \frac{d^{j} u(x)}{d x^{j}}\right\|_{L_{2}(R ; H)} \leq c_{j}\|u\|_{W_{2}^{5}(R ; H)}, j=1,2,3,4,5
$$

are correct.

Equation 1.1 has the following operator form: $Q u(x) \equiv Q_{0} u(x)+Q_{1} u(x)=f(x)$, where $Q_{0}=\left(-\frac{d^{2}}{d x^{2}}+A^{2}\right)\left(\frac{d}{d x}+A\right)^{3}$ and $Q_{1}=\sum_{j=1}^{5} A_{s} \frac{d^{5-j}}{d x^{5-j}}$.

The following theorem provides the association between the norms of operators of intermediate derivatives and the solvability conditions of the problems (1.1) and (1.2).

Theorem 1 The operator $Q_{0}$ isomorphically maps the space $W_{2}^{5}(R ; H)$ onto the space $L_{2}(R ; H)$, moreover, for $f(x) \in L_{2}(R ; H)$ and $E q .1 .1$ has a solution

$$
u(x)=\int_{-\infty}^{+\infty} G(x-s) f(s) d s+u_{0}(x)
$$

where

$$
\begin{aligned}
G(x-s) & =2^{-4} \begin{cases}\left(E+2 A(x-s)+2 A^{2}(x-s)^{2}+\right. & \text { if } x>s, \\
\left.+\frac{8}{6} A^{3}(x-s)^{3}\right) e^{-A(x-s)} A^{-4}, & \text { if } x<s \\
e^{A(x-s)} A^{-4}\end{cases} \\
u_{0}(x) & =-2^{-4}\left(\left(E+2 A x+2 A^{2} x^{2}+\frac{4}{3} A^{3} x^{3}\right) \int_{0}^{\infty} e^{-A(x+s)} A^{-4} f(s) d s+\right. \\
& +\left(E-2 A s+2 A^{2} s^{2}-\frac{4}{3} A^{3} s^{3}\right) \int_{-\infty}^{0} e^{-A(x-s)} A^{-4} f(s) d s+ \\
& +2 A x\left(E-2 A s+2 A^{2} s^{2}\right) \int_{-\infty}^{0} e^{-A(x-s)} A^{-4} f(s) d s+ \\
& +2 A^{2} x^{2}(E-2 A s) \int_{-\infty}^{0} e^{-A(x-s)} A^{-4} f(s) d s+ \\
& \left.+\frac{4}{3} A^{3} x^{3} \int_{-\infty}^{0} e^{-A(x-s)} A^{-4} f(s) d s\right) .
\end{aligned}
$$


Proof Let Eq. 1.1 has a solution $u(x)=u_{1}(x)+u_{0}(x)$, where

$$
u_{1}(x)=\int_{-\infty}^{+\infty} G(x-s) f(s) d s
$$

and

$$
\begin{aligned}
u_{0}(x) & =\phi_{0} e^{-A x}+\phi_{1} A x e^{-A x}+\phi_{2} A^{2} x^{2} e^{-A x}+\phi_{3} A^{3} x^{3} e^{-A x}, \\
\phi_{0} & =-u_{1}(0), \\
\phi_{1} & =\phi_{0}-A^{-1} u_{1}^{\prime}(0), \\
\phi_{2} & =\phi_{1}-\frac{1}{2} \phi_{0}-\frac{1}{2} A^{-2} u_{1}^{\prime \prime}(0), \\
\phi_{3} & =\frac{1}{6} \phi_{0}-\frac{1}{6} A^{-3} u_{1}^{\prime \prime \prime}(0)-\frac{1}{2} \phi_{1}+\phi_{2},
\end{aligned}
$$

First, we find Green's function of Eq. 1.1 where, $A_{j}=0, j=1,2,3,4,5$.

The operator $Q_{0}$ can be simplified on the following form:

$$
Q_{0}=\left(-\frac{d}{d x}+A\right)\left(\frac{d}{d x}+A\right)^{4},
$$

then applying Fourier transform to the equation $Q_{0} u(x)=f(x)$, we obtain:

$$
(-i \xi E+A)(i \xi E+A)^{4} \tilde{u}(\xi)=\tilde{f}(\xi) .
$$

where $E$ - identity operator and $\tilde{u}(\xi), \tilde{f}(\xi)$ are Fourier transforms to the functions $u(x), f(x)$, respectively.

Thus, the polynomial operator pencil $(i \xi E-A)(i \xi E+A)^{4}$ is invertible, and moreover,

$$
\tilde{u}(\xi)=\frac{1}{(-i \xi E+A)(i \xi E+A)^{4}} \tilde{f}(\xi),
$$

hence,

$$
u(x)=\frac{1}{2 \pi} \int_{-\infty}^{+\infty}(-i \xi E+A)^{-1}(i \xi E+A)^{-4} \tilde{f}(\xi) e^{i \zeta(t-s)} d \zeta .
$$

Using Cauchy's theorem of residues,

at $x<s$

$$
\operatorname{Res}=\lim _{i \xi \rightarrow A} \frac{e^{i \zeta(t-s)}}{(i \xi E+A)^{4}}=2^{-4} e^{A(x-s)} A^{-4},
$$

at $x>s$

$$
\begin{aligned}
\operatorname{Res} & =\frac{1}{6} \lim _{i \xi \rightarrow-A} \frac{d^{3}}{d \xi^{3}}\left(\frac{e^{i \zeta(t-s)}}{-i \xi E+A}\right)= \\
& =\left(E+2 A(x-s)+2 A^{2}(x-s)^{2}+\frac{8}{6} A^{3}(x-s)^{3}\right) e^{-A(x-s)} A^{-4} .
\end{aligned}
$$

Then from inequality $(2.1)$, it is simple to prove that $Q_{0}$ which acts from $W_{2}^{5}(R ; H)$ to $L_{2}(R ; H)$ is bounded (see [10]).

Now, we show that $u(x) \in W_{2}^{5}(R ; H)$.

Using the Parseval's equality and (2.2), we obtain:

$$
\begin{aligned}
& \|u\|_{W_{2}^{5}(R ; H)}^{2}=\left\|\frac{d^{5} u}{d x^{5}}\right\|_{L_{2}(R ; H)}^{2}+\left\|A^{5} u\right\|_{L_{2}(R ; H)}^{2}= \\
& =\left\|i \zeta^{5} \tilde{u(\xi)}\right\|_{L_{2}(R ; H)}^{2}+\left\|A^{5} \tilde{u(\xi)}\right\|_{L_{2}(R ; H)}^{2}= \\
& =\left\|i \zeta^{5}(-i \xi E+A)^{-1}(i \xi E+A)^{-4} \tilde{f}(\xi)\right\|_{L_{2}(R ; H)}^{2}+
\end{aligned}
$$




$$
\begin{aligned}
& +\left\|A^{5}(-i \xi E+A)^{-1}(i \xi E+A)^{-4} \tilde{f}(\xi)\right\|_{L_{2}(R ; H)}^{2} \leq \\
& \leq \sup _{\zeta \in R}\left\|i \zeta^{5}(-i \zeta \mathrm{E}+A)^{-1}(i \zeta \mathrm{E}+A)^{-4}\right\|_{H \rightarrow H}^{2}\|\tilde{f}(\zeta)\|_{L_{2}(R ; H)}^{2}+ \\
& +\sup _{\zeta \in R}\left\|A^{5}(-i \zeta \mathrm{E}+A)^{-1}(i \zeta \mathrm{E}+A)^{-4}\right\|_{H \rightarrow H}^{2}\|\tilde{f}(\zeta)\|_{L_{2}(R ; H)}^{2} .
\end{aligned}
$$

From the spectral decomposition of the operator A $(\sigma(\mathrm{A})$ - the spectrum of operator A) for $\zeta \in \mathrm{R}$ (see [11]), we have:

$$
\begin{aligned}
& \left\|i \zeta^{5}(-i \zeta \mathrm{E}+A)^{-1}(i \zeta \mathrm{E}+A)^{-4}\right\|_{H \rightarrow H}=_{\sigma \in \sigma(A)}\left|i \zeta^{5}(-i \zeta+\sigma)^{-1}(i \zeta+\sigma)^{-4}\right| \leq \\
& \leq \sup _{\sigma \in \sigma(A)} \frac{|\zeta|^{5}}{\left(\zeta^{2}+\sigma^{2}\right)^{\frac{5}{2}}} \leq 1, \\
& \left\|A^{5}(-i \zeta \mathrm{E}+A)^{-1}(i \zeta \mathrm{E}+A)^{-4}\right\|_{H \rightarrow H}=\sup _{\sigma \in \sigma(A)}\left|\sigma^{5}(-i \zeta \mathrm{E}+\sigma)^{-1}(i \zeta \mathrm{E}+\sigma)^{-4}\right| \leq \\
& \leq \sup _{\sigma \in \sigma(A)} \frac{\sigma^{5}}{\left(\zeta^{2}+\sigma^{2}\right)^{\frac{5}{2}}} \leq 1 .
\end{aligned}
$$

From (2.4) and (2.5) into (2.3), we obtain:

$$
\|u\|_{W_{2}^{5}(R ; H)}^{2} \leq 2\|\tilde{f}(\zeta)\|_{L_{2}(R ; H)}^{2}=2\|f(x)\|_{L_{2}(R ; H)}^{2} .
$$

Hence, $u(x) \in W_{2}^{5}(R ; H)$.

Using the Banach theorem of the inverse operator, then the operator $Q_{0}$ is an isomorphism from $W_{2}^{5}(R ; H)$ to $L_{2}(R ; H)$ (see [12]).

Before we formulate exact conditions on regular solution of the problems (1.1) and (1.2), expressed only by its operator coefficients, we must estimate the norms of intermediate derivative operators participating in the perturbed part of the given equation. Theorem 1 leads to the norm $\left\|Q_{0} u\right\|_{L_{2}(R ; H)}$ is equivalent to the norm $\|u\|_{W_{2}^{5}(R ; H)}$ in the space $W_{2}^{5}(R ; H)$. Therefore by the norm $\left\|Q_{0} u\right\|_{L_{2}(R ; H)}$, the theorem on intermediate derivatives is valid as well.

Theorem 2 When the function $u(x) \in W_{2}^{5}(R ; H)$, so it keeps the following inequalities:

$$
\left\|A^{j} \frac{d^{5-j} u(x)}{d x^{5-j}}\right\|_{L_{2}(R ; H)} \leq b_{j}\left\|Q_{0} u\right\|_{L_{2}(R ; H)}, j=\overline{1,5}
$$

true, where $b_{1}=b_{4}=\frac{16}{25 \sqrt{5}}, b_{2}=b_{3}=\frac{6 \sqrt{3}}{25 \sqrt{5}}, b_{5}=1$ see [13].

Proof To establish the validity of inequalities (2.6), we take $Q_{0} u(x)=f(x)$ and apply the Fourier transformation as follows:

$$
\begin{aligned}
& \left\|A^{j}(i \zeta)^{5-j}(-i \zeta \mathrm{E}+A)^{-1}(i \zeta \mathrm{E}+A)^{-4} \tilde{f}(\zeta)\right\|_{L_{2}(R ; H)} \leq \\
& \leq \sup _{\zeta \in R}\left\|A^{j}(i \zeta)^{5-j}(-i \zeta \mathrm{E}+A)^{-1}(i \zeta \mathrm{E}+A)^{-4}\right\|_{H \rightarrow H}\|\tilde{f}(\zeta)\|_{L_{2}(R ; H)}, \\
& j=1,2,3,4,5
\end{aligned}
$$

For $\zeta \in R$, we have:

$$
\left\|A^{j}(i \zeta)^{5-j}(-i \zeta \mathrm{E}+A)^{-1}(i \zeta \mathrm{E}+A)^{-4}\right\|_{H \rightarrow H} \leq
$$




$$
\begin{aligned}
& \leq \sup _{\sigma \in \sigma(A)}\left|\sigma^{j}(i \zeta)^{5-j}(-i \zeta \mathrm{E}+\sigma)^{-1}(i \zeta \mathrm{E}+\sigma)^{-4}\right|= \\
& =\sup _{\eta=\frac{\xi^{2}}{\sigma^{2}} \geq 0} \frac{\eta^{((5-j) / 2)}}{(\eta+1)^{\frac{5}{2}}}=b_{j}, j=1,2,3,4,5 .
\end{aligned}
$$

Using inequalities (2.7), we have:

$$
\begin{aligned}
& \left\|A^{j}(i \zeta)^{5-j}(-i \zeta \mathrm{E}+A)^{-1}(i \zeta \mathrm{E}+A)^{-4} \tilde{f(\zeta)}\right\|_{L_{2}(R ; H)} \leq \\
& \leq b_{j}\|\tilde{f(\xi)}\|_{L_{2}(R ; H)}, j=1,2,3,4,5 .
\end{aligned}
$$

Lemma 1 The operator $Q_{1}$ continuously acts from $W_{2}^{5}(R ; H)$ to $L_{2}(R ; H)$ provided that the operators $A_{j} A^{-j}, j=1,2,3,4,5$ are bounded in $H$.

Considering the results found up to now see [14], for problems (1.1) and (1.2), we get the possibility to establish regular solvability conditions.

Theorem 3 Let $|\kappa|<2 \lambda_{0}\left(A=A * \geq \lambda_{o} E, \lambda_{o}>0\right)$ for any $u(t) \in W_{2}^{5}(R ; H)$, then holds the inequality

$$
\begin{aligned}
& \qquad \begin{array}{l}
b(k)\left(c_{1}(k)\left\|A_{1} A^{-1}\right\|_{H \rightarrow H}+c_{2}(k)\left\|A_{2} A^{-2}\right\|_{H \rightarrow H}+c_{3}(k)\left\|A_{3} A^{-3}\right\|_{H \rightarrow H}+\right. \\
\left.+c_{4}(k)\left\|A_{4} A^{-4}\right\|_{H \rightarrow H}\right)<1 \text { see }[15], \\
\text { where } \\
\qquad c_{1}(k)=\left[1+\frac{4 \lambda_{0}\left|\lambda_{0}+k\right|}{\left(2 \lambda_{0}+k\right)^{2}}\right]^{\frac{3}{2}}, c_{2}(k)=\frac{2 \lambda_{0}}{2 \lambda_{0}+k}\left[1+\frac{4 \lambda_{0}\left|\lambda_{0}+k\right|}{\left(2 \lambda_{0}+k\right)^{2}}\right] \\
\quad c_{3}(k)=\frac{4 \lambda_{0}^{2}}{\left(2 \lambda_{0}+k\right)^{2}}\left[1+\frac{4 \lambda_{0}\left|\lambda_{0}+k\right|}{\left(2 \lambda_{0}+k\right)^{2}}\right]^{\frac{1}{2}}, c_{4}(k)=\frac{8 \lambda_{0}^{3}}{\left(2 \lambda_{0}+k\right)^{3}},
\end{array}
\end{aligned}
$$

and

$$
b(k)= \begin{cases}\frac{\lambda_{0}}{2^{\frac{1}{2}}\left(2 \lambda_{0}^{2}-k^{2}\right)^{\frac{1}{2}}}, & \text { if } 0 \leq \frac{k^{2}}{4 \lambda_{0}^{2}}<\frac{1}{3}, \\ \frac{2 \lambda_{0}|k|}{4 \lambda_{0}^{2}-k^{2}}, & \text { if } \frac{1}{3} \leq \frac{k^{2}}{4 \lambda_{0}^{2}}<1 .\end{cases}
$$

Theorem 4 Suppose that the operators $A_{j} A^{-j}, j=\overline{1,4}$, be bounded in $H$ and they hold the inequality

$$
\sum_{j=1}^{4} C_{j}(k) b(k)\left\|A_{j} A^{-j}\right\|_{H \rightarrow H}<1,
$$

where the numbers $C_{j}(k), j=1,2,3,4$ and $b(k)$ are determined in Theorem 3 so the problems (1.1) and (1.2) are regularly solvable (see $[16,17])$.

Proof where $f(x) \in L_{2}(R ; H), u(x) \in W_{2}^{5}(R ; H)$ and by Theorem (1.1), there exists a bounded inverse operator to $Q_{0}$, which acts from $L_{2}(R ; H)$ to $W_{2}^{5}(R ; H)$; then after replacing $Q_{0} u(x)=w(x)$ in Eq. 1.1, it can be written as $\left(E+Q_{1} Q_{0}^{-1}\right) w(x)=f(x)$.

Now, we prove under the theorem conditions see [18], that

$$
\left\|Q_{1} Q_{0}^{-1}\right\|_{L_{2}(R ; H) \rightarrow L_{2}(R ; H)}<1 .
$$

By Theorem 3, we have:

$$
\begin{aligned}
& \left\|Q_{1} Q_{0}^{-1} w\right\|_{L_{2}(R ; H)}=\left\|Q_{1} u\right\|_{L_{2}(R ; H)} \leq \sum_{j=1}^{4}\left\|A_{j} \frac{d^{4-j} u_{u}}{d x^{4-j}}\right\|_{L_{2}(R ; H)} \leq \\
& \leq \sum_{j=1}^{4}\left\|A_{j} A^{-j}\right\|_{H \rightarrow H}\left\|A^{j} \frac{d^{4-j} u}{d x^{4-j}}\right\|_{L_{2}(R ; H)} \leq
\end{aligned}
$$




$$
\begin{aligned}
& \leq \sum_{j=1}^{4} C_{j}(k) b(k)\left\|A_{j} A^{-j}\right\|_{H \rightarrow H}\left\|Q_{0} u\right\|_{L_{2}(R ; H)}= \\
& =\sum_{j=1}^{4} C_{j}(k) b(k)\left\|A_{j} A^{-j}\right\|_{H \rightarrow H}\|v\|_{L_{2}(R ; H)} .
\end{aligned}
$$

Consequently,

$$
\left\|Q_{1} Q_{0}^{-1}\right\|_{L_{2}(R ; H) \rightarrow L_{2}(R ; H)} \leq \sum_{j=1}^{4} C_{j}(k) b(k)\left\|A_{j} A^{-j}\right\|_{H \rightarrow H}<1 \text {. Thus, the oper- }
$$
ator $E+Q_{1} Q_{0}^{-1}$ is invertible in $L_{2}(R ; H)$; therefore, $u(x)$ can be determined by $u(x)=Q_{0}^{-1}\left(E+Q_{1} Q_{0}^{-1}\right)^{-1} f(x) ;$ moreover:

$$
\begin{aligned}
& \|u\|_{W_{2}^{5}(R ; H)} \leq\left\|Q_{0}^{-1}\right\|_{L_{2}(R ; H) \rightarrow W_{2}^{5}(R ; H)} \times\left\|\left(\left(E+Q_{1} Q_{0}^{-1}\right)\right)^{-1}\right\|_{L_{2}(R ; H) \rightarrow L_{2}(R ; H)}\|f\|_{L_{2}(R ; H)} \\
& \leq \text { const }\|f\|_{L_{2}(R ; H)}
\end{aligned}
$$

\section{Conclusion}

In the whole real axis, with a multiple characteristic, fifth-order and self-adjoint differential operator has not been researched so far. We demonstrated the association between the coefficients of the differential operator and the conditions of problems (1.1) and (1.2) to be regularly solvable. We estimated the norms of intermediate derivative operators which appear in the essential part of the investigated equation, and we proved that the maximum value of the $b_{j}, j=1,2,3,4,5$ does not exceed one. The norms of the linear operators participating in the second part are estimated and used to formulate the exact solvability conditions.

\section{Acknowledgements}

We thank our colleagues from Ain Shams University who provided insight and expertise that greatly assisted the research.

Authors' contributions

The authors contributed equally to this work. All authors read and approved the final manuscript.

\section{Funding}

Not applicable.

Availability of data and materials

Not applicable.

Competing interests

The authors declare that they have no competing interests.

\section{Author details}

${ }^{1}$ Ain Shams University, 11865 Cairo, Egypt. ${ }^{2}$ Helwan University, 11865 Cairo, Egypt. ${ }^{3}$ Egyptian Russian University, 11865 Cairo, Egypt.

Received: 3 April 2019 Accepted: 7 August 2019

Published online: 21 October 2019

\section{References}

1. Markus, H.: Functional analysis: an elementary introduction. Am. Math. Soc., 394 (1970)

2. Lions, J. L., Majenes, E.: Non-homogeneous boundary value problems and their applications. Springer-Verlag Berlin, Heidelberg (1972)

3. Bitsadze, A. V.: Boundary Value Problems For Second Order Elliptic Equations, North Holland (1968)

4. Krein, S. G.: Linear differential equations in a banach space, Nauka (1967)

5. Yakubov, S, Ya: Linear differential operator equations and their applications. Baku, "Elm", Russian (1985)

6. Aliev, A. R., Gasymov, A. A.: On the Correct Solvability of the Boundary-Value Problem for One Class Operator-Differential Equations of the Fourth order with Complex Characteristics. Bound. Value Probl. 2009, 1-20 (2009)

7. Aliev, A. R., Lachinova, F. S.: On the solvability in a weighted space of an initial boundary value problem for a third order operator differential equation with a parabolic principal part. Dokl. Math. 93(1), 85-88 (2016)

8. Lachinova, F. S.: Solvability of a class of parabolic operator-differentia Equations of third order. Proc. IMM NAS Azerbaijan XXXIX, 77-86 (2013)

9. Brezis, H.: Functional analysis, Sobolev spaces and partial differential equations, USA (2011) 
10. Aliev, A. R., Soylemezo, M. A.: Problem without initial conditions for a class of inverse parabolic operator differential equations of third order. Dokl. Math. 97(3), 199-202 (2018)

11. Aliev, A. R., Mirzoev, S. S., Soylemezo, M. A.: On solvability of third order operator differential equation with parabolic principal part in weigted space. J. Funct. Spaces. 2017, 1-8 (2017)

12. Al-Aidarous, E. S, Aleiv, A. R., Razayev, E. S., Zedan, H. A.: Fourth order elliptic operator-differential equations with unbounded operator boundary conditions in the Sobolev-type spaces. Bound. Value Probl., 1-14 (2015)

13. Abdel Baset, I., Ahmed Labeeb, M. A.: Solvability of a Class of Operator-Differential Equations of Third Order with Complicated Characteristic on the Whole Real Axis. Open Access Libr. J. 5 (2018)

14. Aliev, A. R., Elbably, A. L.: On the solvability in a weight space of a third-order operator-differential equation with multiple characteristic. Dokl. Math. 85(2), 233-235 (2012)

15. Aliev, A. R.: On the solvability of a class of operator differential equations of the second order on the real axis. J. Math. Phys. Anal. Geom. 2(4), 347-357 (2006)

16. Aliev, A. R., Mohamed, A. S.: On the well-posed of a boundary value problem for a class of fourth order operator differential equations. Differ. Equ. 48(4), 596-598 (2012)

17. Aliev, A. R., Elbably, A. L.: Well-posedness of a boundary value problem for a class of third-order operator-differential equations. Bound. Value Probl., 1-15 (2013)

18. Aliev, A. R.: On the solvability of the equations containing in the main part the operators of the form $-\frac{d^{3}}{d t^{3}}+A^{3}$ in the weight space. Trans. NAS Azerbaijan, 9-16 (2006)

\section{Publisher's Note}

Springer Nature remains neutral with regard to jurisdictional claims in published maps and institutional affiliations.

\section{Submit your manuscript to a SpringerOpen ${ }^{\circ}$ journal and benefit from:}

- Convenient online submission

Rigorous peer review

Open access: articles freely available online

- High visibility within the field

- Retaining the copyright to your article

Submit your next manuscript at $\boldsymbol{\triangleright}$ springeropen.com 